\title{
An Efficient Method for the Hydrolysis of Potassium Organotrifluoroborates Promoted by Montmorillonite K10
}

\author{
Renato L. Silva, ${ }^{a}$ Cosme S. Santos, ${ }^{b}$ Jonh A. M. Santos, ${ }^{b}$ Roberta A. Oliveira, \\ Paulo H. Menezes ${ }^{a}$ and Juliano C. R. Freitas*,b,c \\ ${ }^{a}$ Departamento de Química Fundamental, Universidade Federal de Pernambuco, \\ 50740-560 Recife-PE, Brazil \\ ${ }^{b}$ Departamento de Química, Universidade Federal Rural de Pernambuco, \\ 52171-900 Recife-PE, Brazil \\ ${ }^{c}$ Centro de Educação e Saúde, Universidade Federal de Campina Grande, \\ 58175-000 Cuité-PB, Brazil
}

\begin{abstract}
An efficient and non-expensive method for conversion of diverse potassium organotrifluoroborates to their corresponding boronic acids promoted by montmorillonite K10 using water as the reaction solvent is described. Further interconversion of potassium organotrifluoroborates to their corresponding boronic esters, via boronic acid intermediates was also successfully accomplished. The products were obtained in good yields, being the rate of hydrolysis influenced by the type of substituent present in the boronic acid.
\end{abstract}

Keywords: potassium organotrifluoroborates, boronic acids, boronic esters, montmorillonite K10

\section{Introduction}

Transition metal-catalyzed cross-coupling reaction between electrophilic reagents and organometallic compounds are currently the most used method for the formation of new $\mathrm{C}-\mathrm{C}$ bonds. ${ }^{1}$ Although a wide variety of organometallic reagents can be used, all with their advantages and limitations, the Suzuki reaction has become the most used due to the mild conditions required to be performed, relative stability and low toxicity of boronic acids. $^{2}$

Boronic acids are the most common partner in Suzuki cross-coupling reactions due to the high Lewis acidity of boron atom, since this is the critical factor of the transmetalation step in the mechanism. In addition, these compounds have also been used as drug delivery agents, ${ }^{3}$ sensors, ${ }^{4}$ as well as biological inhibitors. ${ }^{5}$ Moreover, the recent interest in small molecules containing boronic acids, in special after the Food and Drug Administration (FDA) approval of the drug Velcade, makes boronic acids compounds of great interest.

*e-mail: julianocrufino@pq.cnpq.br
These reagents, however, have some limitations: many are difficult to purify, and are in equilibrium with anhydrides (boroximes), which makes determination of the stoichiometry of the reaction also difficult. As a consequence, they are generally used in excess. ${ }^{6}$ Some of these problems can be solved by the use of boronic esters, which exist only in the monomeric form. ${ }^{7}$ However, the low atom economy and the high cost of pinacol make the use of these reagents unattractive. ${ }^{8}$

On the other hand, transmetalation is sometimes not desired and the maintenance of the boron atom to be used for further reactions, preferably in an orthogonal manner, is a subject of great interest. ${ }^{9}$ Within this context, some boron protecting groups such as $N$-methyliminodiacetyl $[\mathrm{B}(\text { mida })]^{10}$ and 1,8 -naphthalenediamine $[\mathrm{B}(\mathrm{dan})]^{11}$ were developed, however, despite easy removal under mild conditions, the insertion of these groups requires longer reaction times and high temperatures.

Potassium organotrifluoroborates emerged as an alternative to $[\mathrm{B}($ mida $)]$ and $[\mathrm{B}(\mathrm{dan})]$ as a protecting group for boronic acids ${ }^{12}$ specially due to their stability and crystallinity. Moreover, organotrifluoroborates are the most atom-efficient boronic acid surrogates available. ${ }^{13} \mathrm{In}$ 
addition, some methods are available to hydrolyze these reagents back into the corresponding boronic acids or boronates. ${ }^{14}$ Indeed, it is also known that the hydrolysis of potassium organotrifluoroborates is very dependent on the organic moiety. ${ }^{15}$

Additionally to these characteristics, the reactivity differences between organotrifluoroborates and boranes in some dibora intermediates ${ }^{16}$ can be explored in the crosscoupling reactions in a highly chemoselective fashion, leaving the trifluoroborate moiety intact for subsequent transformations.

Within this context, this work describes an efficient method for conversion of potassium organotrifluoroborates into the corresponding boronic acids or esters using montmorillonite K10 under green conditions.

\section{Experimental}

All reagents and solvents used (purchased from Aldrich Chemical Co.) were previously purified and dried in agreement with the literature, ${ }^{17}$ and used as received. Reactions were monitored by thin-layer chromatography on $0.25 \mathrm{~mm}$ E. Merck silica gel 60 plates (F254) using UV light, vanillin and $p$-anisaldehyde as visualizing agents.
${ }^{1} \mathrm{H}$ and ${ }^{13} \mathrm{C}$ nuclear magnetic resonance (NMR) data were recorded in $\mathrm{CDCl}_{3}$ or dimethylsulfoxide- $d_{6}$ (DMSO- $d_{6}$ ). The chemical shifts are reported as delta $(\delta)$ units in parts per million (ppm) relative to the solvent residual peak as the internal reference. ${ }^{11} \mathrm{~B}(128 \mathrm{MHz}) \mathrm{NMR}$ spectra and ${ }^{19} \mathrm{~F}(376 \mathrm{MHz})$ were calibrated using $\mathrm{BF}_{3} \cdot \mathrm{Et}_{2} \mathrm{O}(0.0 \mathrm{ppm})$ as external reference in the case of ${ }^{11} \mathrm{~B}$ NMR and chemical shifts were referenced to external $\mathrm{CF}_{3} \mathrm{CO}_{2} \mathrm{H}(0.0 \mathrm{ppm})$ in the case of ${ }^{19} \mathrm{~F}$ NMR. Coupling constants $(J)$ for all spectra are reported in hertz $(\mathrm{Hz})$.

General procedure for the hydrolysis of potassium organotrifluoroborates (2a-20)

In a flask containing the appropriate potassium organotrifluoroborate $(0.5 \mathrm{mmol})$ in distilled water $(1 \mathrm{~mL})$ was added montmorillonite $\mathrm{K} 10(150 \% \mathrm{~m} / \mathrm{m})$. The mixture was stirred for the time indicated in Scheme 1 at room temperature. After this period, the mixture was extracted with EtOAc $(3 \times 10 \mathrm{~mL})$ and the organic phase was washed with water $(2 \times 15 \mathrm{~mL})$. The organic phase was dried over anhydrous $\mathrm{MgSO}_{4}$, filtered and the solvent was removed in vacuo to yield the corresponding boronic acids $\mathbf{2 a - 0 .}$

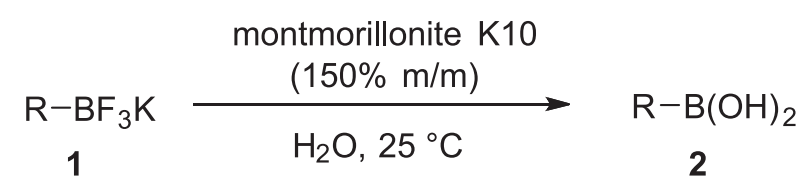<smiles>Oc1ccccc1</smiles>

$2 a$ $97 \%, 30 \mathrm{~min}$<smiles>COc1ccc(Br)cc1</smiles>

2b (o) $80 \%, 3 \mathrm{~h}$ 2c $(m) 85 \%, 2 \mathrm{~h}$ 2d $(p) 90 \%, 1.5 \mathrm{~h}$<smiles>[X]c1ccc(Br)cc1</smiles>

2g $(X=F) 84 \%, 20 \mathrm{~min}$ 2h $(X=B r) 74 \%, 45$ min<smiles>O=Cc1ccc(Br)cc1</smiles>

2i

$75 \%, 24 \mathrm{~h}$<smiles>Oc1ccc(Br)cc1</smiles>

$87 \%, 1.5 \mathrm{~h}$<smiles>[X]c1cccc([X])c1Br</smiles>

2j $(X=M e) 95 \%, 20 \mathrm{~min}$ 2k $(X=F) 81 \%, 1 \mathrm{~h}$<smiles>Nc1cccc(Br)c1</smiles>
$78 \%, 1.5 \mathrm{~h}$<smiles>Oc1ccsc1</smiles>

$2 \mathrm{~m}$

$96 \%, 45 \mathrm{~min}$<smiles>Oc1ccc2oc3ccccc3c2c1</smiles>

$86 \%, 75 \mathrm{~min}$<smiles>OCCc1ccccc1</smiles>

20 $86 \%, 1 \mathrm{~h}$<smiles>O=Cc1ccc(F)c(Br)c1</smiles>

2I $90 \%, 24 \mathrm{~h}$<smiles>C=C=C[Hg]O</smiles>

$2 p$

Scheme 1. Hydrolysis of different potassium organotrifluoroborates to the corresponding boronic acids using montmorillonite K10. 
Phenylboronic acid (2a)

Yield: $59 \mathrm{mg}(97 \%)$ as a white solid; $\mathrm{mp}>200{ }^{\circ} \mathrm{C}$; ${ }^{1} \mathrm{H}$ NMR (400 MHz, DMSO- $\left.d_{6}\right) \delta 8.03\left(\mathrm{~s}, 2 \mathrm{H}, \mathrm{B}(\mathrm{OH})_{2}\right)$, 7.81 (dd, $J 8.0$ and $\left.1.2 \mathrm{~Hz}, 2 \mathrm{H}, \mathrm{H}_{\text {aryl }}\right), 7.41-7.31(\mathrm{~m}, 3 \mathrm{H}$, $\left.\mathrm{H}_{\text {aryl }}\right) ;{ }^{13} \mathrm{C}$ NMR (100 MHz, DMSO- $\left.d_{6}\right) \delta 134.1,130.1$, $127.4 ;{ }^{11} \mathrm{~B}$ NMR $\left(128 \mathrm{MHz}, \mathrm{CDCl}_{3}\right) \delta 28.3$ (s). This is in agreement with data previously reported. ${ }^{14}$

\section{2-Methoxyphenylboronic acid (2b)}

Yield: $61 \mathrm{mg}(80 \%)$; white solid; $\mathrm{mp}>200{ }^{\circ} \mathrm{C} ;{ }^{1} \mathrm{H}$ NMR $\left(400 \mathrm{MHz}, \mathrm{DMSO}-d_{6}\right) \delta 7.69\left(\mathrm{~s}, 2 \mathrm{H}, \mathrm{B}(\mathrm{OH})_{2}\right), 7.60(\mathrm{dd}$, $J 7.2$ and $\left.2.0 \mathrm{~Hz}, 1 \mathrm{H}, \mathrm{H}_{\text {aryl }}\right), 7.36(\mathrm{td}, J 7.2$ and $2.0 \mathrm{~Hz}, 1 \mathrm{H}$, $\left.\mathrm{H}_{\text {aryl }}\right), 6.97-6.92\left(\mathrm{~m}, 2 \mathrm{H}, \mathrm{H}_{\text {aryl }}\right), 3.81$ (s, 3H, OMe $) ;{ }^{13} \mathrm{C} \mathrm{NMR}$ $\left(100 \mathrm{MHz}, \mathrm{DMSO}-d_{6}\right) \delta 163.6,136.4,131.7,120.3,110.3$, $55.3 ;{ }^{11} \mathrm{~B}$ NMR $\left(128 \mathrm{MHz}, \mathrm{DMSO}-d_{6}\right) \delta 28.7$ (s). This is in agreement with data previously reported. ${ }^{14}$

\section{3-Methoxyphenylboronic acid (2c)}

Yield: $65 \mathrm{mg}$ (85\%); white solid; $\mathrm{mp}>200{ }^{\circ} \mathrm{C} ;{ }^{1} \mathrm{H}$ NMR $\left(400 \mathrm{MHz}, \mathrm{DMSO}-d_{6}\right) \delta 8.04\left(\mathrm{~s}, 2 \mathrm{H}, \mathrm{B}(\mathrm{OH})_{2}\right), 7.38-7.36$ $\left(\mathrm{m}, 2 \mathrm{H}, \mathrm{H}_{\text {aryl }}\right), 7.25\left(\mathrm{dd}, J 8.4\right.$ and $\left.7.2 \mathrm{~Hz}, 1 \mathrm{H}, \mathrm{H}_{\text {aryl }}\right)$, 6.97-6.94 (m, 1H, $\left.\mathrm{H}_{\text {aryl }}\right), 3.74$ (s, 3H, OMe); ${ }^{13} \mathrm{C}$ NMR $\left(100 \mathrm{MHz}, \mathrm{DMSO}-d_{6}\right) \delta 158.6,128.6,126.4,119.1,115.8$, $54.9 ;{ }^{11} \mathrm{~B}$ NMR $\left(128 \mathrm{MHz}, \mathrm{DMSO}-d_{6}\right) \delta 29.2$ (s). This is in agreement with data previously reported. ${ }^{14,18}$

\section{4-Methoxyphenylboronic acid (2d)}

Yield: $68.5 \mathrm{mg}$ (90\%); white solid; $\mathrm{mp}>200{ }^{\circ} \mathrm{C}$; ${ }^{1} \mathrm{H}$ NMR (400 MHz, DMSO- $\left.d_{6}\right) \delta 7.84$ (s, 2H, B(OH) $)$ ), $7.75\left(\mathrm{~d}, J 8.4 \mathrm{~Hz}, 2 \mathrm{H}, \mathrm{H}_{\text {aryl }}\right), 6.89\left(\mathrm{~d}, J 8.8 \mathrm{~Hz}, 2 \mathrm{H}, \mathrm{H}_{\text {aryl }}\right), 3.75$ $(\mathrm{s}, 3 \mathrm{H}, \mathrm{OMe}) ;{ }^{13} \mathrm{C}$ NMR (100 MHz, DMSO- $\left.d_{6}\right) \delta 161.0$, 135.9, 112.9, 54.9; ${ }^{11} \mathrm{~B}$ NMR (128 MHz, DMSO- $\left.d_{6}\right) \delta 28.7$ (s). This is in agreement with data previously reported. ${ }^{14}$

\section{4-Hydroxyphenylboronic acid (2e)}

Yield: $60 \mathrm{mg}$ (87\%); white solid; $\mathrm{mp} 184-186{ }^{\circ} \mathrm{C}$; ${ }^{1} \mathrm{H}$ NMR (400 MHz, DMSO- $\left.d_{6}\right) \delta 9.51(\mathrm{~s}, 1 \mathrm{H}, \mathrm{OH}), 7.71$ (s, $\left.2 \mathrm{H}, \mathrm{B}(\mathrm{OH})_{2}\right), 7.63\left(\mathrm{~d}, J 8.4 \mathrm{~Hz}, 2 \mathrm{H}, \mathrm{H}_{\text {aryl }}\right), 6.72(\mathrm{~d}, J 8.4 \mathrm{~Hz}$, $\left.2 \mathrm{H}, \mathrm{H}_{\text {ary }}\right) ;{ }^{13} \mathrm{C}$ NMR $\left(100 \mathrm{MHz}, \mathrm{DMSO}-d_{6}\right) \delta 159.3,136.0$, 114.5 ; ${ }^{11} \mathrm{~B}$ NMR $\left(128 \mathrm{MHz}, \mathrm{DMSO}-d_{6}\right) \delta 29.7$ (s). This is in agreement with data previously reported. ${ }^{14,18}$

\section{3-Aminophenylboronic acid (2f)}

Yield: $53 \mathrm{mg}$ (78\%); white solid; mp 142-144 ${ }^{\circ} \mathrm{C}$; ${ }^{1} \mathrm{H} \mathrm{NMR}\left(400 \mathrm{MHz}, \mathrm{CDCl}_{3}\right) \delta 7.75$ (s, 2H, B(OH) $)$, 6.99$6.94\left(\mathrm{~m}, 2 \mathrm{H}, \mathrm{H}_{\text {aryl }}\right), 6.61-6.58\left(\mathrm{~m}, 1 \mathrm{H}, \mathrm{H}_{\text {aryl }}\right), 6.52(\mathrm{~s}, 1 \mathrm{H}$, $\left.\mathrm{H}_{\text {aryl }}\right), 4.91\left(\mathrm{~s}, 2 \mathrm{H}, \mathrm{NH}_{2}\right) ;{ }^{13} \mathrm{C}$ NMR (100 MHz, DMSO- $\left.d_{6}\right)$ $\delta 147.4,127.8,122.0,120.0,115.8 ;{ }^{11} \mathrm{~B}$ NMR $(128 \mathrm{MHz}$, DMSO- $\left.d_{6}\right) \delta 30.2(\mathrm{~s})$. This is in agreement with data previously reported. ${ }^{19}$

\section{4-Fluorophenylboronic acid (2g)}

Yield: $59 \mathrm{mg}$ (84\%); white solid; $\mathrm{mp}>200{ }^{\circ} \mathrm{C} ;{ }^{1} \mathrm{H}$ NMR $\left(400 \mathrm{MHz}, \mathrm{DMSO}-d_{6}\right) \delta 8.07$ (s, 2H, B(OH) $), 7.83$ (dd, $J 8.4$ and $\left.6.4 \mathrm{~Hz}, 2 \mathrm{H}, \mathrm{H}_{\text {aryl }}\right), 7.14\left(\mathrm{t}, J 9.2 \mathrm{~Hz}, 2 \mathrm{H}, \mathrm{H}_{\text {aryl }}\right)$; ${ }^{13} \mathrm{C}$ NMR $\left(100 \mathrm{MHz}, \mathrm{DMSO}-d_{6}\right) \delta 163.8\left(\mathrm{~d}, J_{\mathrm{C}-\mathrm{F}} 244.4 \mathrm{~Hz}\right.$, $\left.\mathrm{C}_{\text {aryl }}\right), 136.5\left(\mathrm{~d}, J_{\mathrm{C}-\mathrm{F}} 8.4 \mathrm{~Hz}, \mathrm{C}_{\text {aryl }}\right), 114.2\left(\mathrm{~d}, J_{\mathrm{C}-\mathrm{F}} 19.7 \mathrm{~Hz}\right.$, $\left.\mathrm{C}_{\text {aryl }}\right) ;{ }^{11} \mathrm{~B}$ NMR $\left(128 \mathrm{MHz}, \mathrm{DMSO}-d_{6}\right) \delta 28.9(\mathrm{~s}) ;{ }^{19} \mathrm{~F} \mathrm{NMR}$ $\left(376 \mathrm{MHz}, \mathrm{DMSO}-d_{6}\right) \delta-100.7(\mathrm{~s})$. This is in agreement with data previously reported. ${ }^{14,18}$

\section{4-Bromophenylboronic acid (2h)}

Yield: $74 \mathrm{mg}$ (74\%); white solid; $\mathrm{mp}>200{ }^{\circ} \mathrm{C}$; ${ }^{1} \mathrm{H}$ NMR (400 MHz, DMSO- $\left.d_{6}\right) \delta 8.15\left(\mathrm{~s}, 2 \mathrm{H}, \mathrm{B}(\mathrm{OH})_{2}\right), 7.72(\mathrm{~d}$, $\left.J 8.4 \mathrm{~Hz}, 2 \mathrm{H}, \mathrm{H}_{\text {aryl }}\right), 7.53\left(\mathrm{~d}, J 8.4 \mathrm{~Hz}, 2 \mathrm{H}, \mathrm{H}_{\text {aryl }}\right) ;{ }^{13} \mathrm{C} \mathrm{NMR}$ $\left(100 \mathrm{MHz}, \mathrm{DMSO}-d_{6}\right) \delta 136.2,130.3,124.1 ;{ }^{11} \mathrm{~B}$ NMR $\left(128 \mathrm{MHz}, \mathrm{DMSO}-d_{6}\right) \delta 29.5(\mathrm{~s})$. This is in agreement with data previously reported. ${ }^{14}$

\section{4-Formylphenylboronic acid (2i)}

Yield: $56 \mathrm{mg}$ (75\%); white solid; $\mathrm{mp}>200{ }^{\circ} \mathrm{C}$; ${ }^{1} \mathrm{H}$ NMR (400 MHz, DMSO- $d_{6}$ ) $\delta 10.02$ (s, 1H, -CHO), 8.34 (s, 2H, $\left.\mathrm{B}(\mathrm{OH})_{2}\right), 7.98\left(\mathrm{~d}, J 7.6 \mathrm{~Hz}, 2 \mathrm{H}, \mathrm{H}_{\text {aryl }}\right), 7.86(\mathrm{~d}, J 8.0 \mathrm{~Hz}$, $\left.2 \mathrm{H}, \mathrm{H}_{\text {aryl }}\right) ;{ }^{13} \mathrm{C}$ NMR $\left(100 \mathrm{MHz}, \mathrm{DMSO}-d_{6}\right) \delta 193.5,137.1$, 134.5, 128.3; ${ }^{11} \mathrm{~B}$ NMR (128 MHz, DMSO- $\left.d_{6}\right) \delta 28.3$ (s). This is in agreement with data previously reported. ${ }^{19}$

\section{(2,6-Dimethylphenyl)boronic acid (2j)}

Yield: $71 \mathrm{mg}$ (95\%); white solid; mp 120-121 ${ }^{\circ} \mathrm{C}$; ${ }^{1} \mathrm{H}$ NMR (400 MHz, DMSO- $\left.d_{6}\right) \delta 8.12\left(\mathrm{~s}, 2 \mathrm{H}, \mathrm{B}(\mathrm{OH})_{2}\right)$, $7.06\left(\mathrm{t}, J 7.2 \mathrm{~Hz}, 1 \mathrm{H}, \mathrm{H}_{\text {aryl }}\right), 6.91\left(\mathrm{~d}, J 7.2 \mathrm{~Hz}, 2 \mathrm{H}, \mathrm{H}_{\text {aryl }}\right), 2.26$ $\left(\mathrm{s}, 6 \mathrm{H},-\mathrm{CH}_{3}\right) ;{ }^{13} \mathrm{C}$ NMR (100 MHz, DMSO- $\left.d_{6}\right) \delta 138.4$, 127.3, 125.6, 21.9; ${ }^{11} \mathrm{~B}$ NMR (128 MHz, DMSO- $\left.d_{6}\right) \delta 32.3$ (s). This is in agreement with data previously reported. ${ }^{14}$

\section{(2,6-Difluorophenyl)boronic acid (2k)}

Yield: $64 \mathrm{mg}(81 \%)$; white solid; $\mathrm{mp}>200{ }^{\circ} \mathrm{C}$; ${ }^{1} \mathrm{H}$ NMR $\left(400 \mathrm{MHz}, \mathrm{DMSO}-d_{6}\right) \delta 8.68\left(\mathrm{~s}, 2 \mathrm{H}, \mathrm{B}(\mathrm{OH})_{2}\right), 7.44-7.36$ $\left(\mathrm{m}, 1 \mathrm{H}, \mathrm{H}_{\text {aryl }}\right), 7.00-6.94\left(\mathrm{~m}, 2 \mathrm{H}, \mathrm{H}_{\text {aryl }}\right) ;{ }^{13} \mathrm{C} \mathrm{NMR}(100 \mathrm{MHz}$, DMSO- $\left.d_{6}\right) \delta 164.0\left(\mathrm{dd}, J_{\mathrm{C}-\mathrm{F}} 294.6\right.$ and $15.2 \mathrm{~Hz}, \mathrm{C}_{\text {aryl }}$ ), $131.3\left(\mathrm{t}, J_{\mathrm{C}-\mathrm{F}} 9.9 \mathrm{~Hz}, \mathrm{C}_{\text {aryl }}\right), 110.8\left(\mathrm{dd}, J_{\mathrm{C}-\mathrm{F}} 20.5\right.$ and $6.8 \mathrm{~Hz}$, $\left.\mathrm{C}_{\text {aryl }}\right) ;{ }^{11} \mathrm{~B}$ NMR $\left(128 \mathrm{MHz}, \mathrm{DMSO}-d_{6}\right) \delta 29.3(\mathrm{~s}) ;{ }^{19} \mathrm{~F} \mathrm{NMR}$ $\left(376 \mathrm{MHz}, \mathrm{DMSO}-d_{6}\right) \delta-103.1$ (s). This is in agreement with data previously reported. ${ }^{14}$

\section{(2-Fluoro-5-formylphenyl)boronic acid (2I)}

Yield: $76 \mathrm{mg}$ (90\%); white solid; $\mathrm{mp}>200{ }^{\circ} \mathrm{C}$; ${ }^{1} \mathrm{H}$ NMR (400 MHz, DMSO-d $)_{6} \delta 9.98$ (s, 1H, -CHO), $8.46\left(\mathrm{~s}, 2 \mathrm{H}, \mathrm{B}(\mathrm{OH})_{2}\right), 8.14\left(\mathrm{dd}, J 6.0\right.$ and $\left.2.4 \mathrm{~Hz}, 1 \mathrm{H}, \mathrm{H}_{\text {aryl }}\right)$, 7.99-7.95 (m, 1H, $\left.\mathrm{H}_{\text {aryl }}\right), 7.32(\mathrm{dd}, J 8.8$ and $8.4 \mathrm{~Hz}, 1 \mathrm{H}$, $\left.\mathrm{H}_{\text {aryl }}\right) ;{ }^{13} \mathrm{C}$ NMR (100 MHz, DMSO- $\left.d_{6}\right) \delta 191.9,168.9(\mathrm{~d}$, $\left.J_{\text {C-F }} 252.7 \mathrm{~Hz}, \mathrm{C}_{\text {aryl }}\right), 137.8\left(\mathrm{~d}, J_{\mathrm{C}-\mathrm{F}} 11.4 \mathrm{~Hz}, \mathrm{C}_{\text {aryl }}\right), 133.3$ 
$\left(\mathrm{d}, J_{\mathrm{C}-\mathrm{F}} 9.9 \mathrm{~Hz}, \mathrm{C}_{\text {ary }}\right), 132.3\left(\mathrm{~d}, J_{\mathrm{C}-\mathrm{F}} 2.2 \mathrm{~Hz}, \mathrm{C}_{\text {ary }}\right), 116.1(\mathrm{~d}$, $\left.J_{\mathrm{C}-\mathrm{F}} 25.8 \mathrm{~Hz}, \mathrm{C}_{\text {ary }}\right) ;{ }^{11} \mathrm{~B}$ NMR $\left(128 \mathrm{MHz}\right.$, DMSO- $\left.d_{6}\right) \delta 30.2$ $(\mathrm{s}) ;{ }^{19} \mathrm{~F}$ NMR $\left(376 \mathrm{MHz}, \mathrm{DMSO}-d_{6}\right) \delta-94.4$ (s). This is in agreement with data previously reported. ${ }^{14,20}$

\section{3-Thienylboronic acid (2m)}

Yield: $61.5 \mathrm{mg}$ (96\%); white solid; mp $132-134{ }^{\circ} \mathrm{C}$; ${ }^{1} \mathrm{H}$ NMR (400 MHz, DMSO- $\left.d_{6}\right) \delta 7.99\left(\mathrm{~s}, 2 \mathrm{H}, \mathrm{B}(\mathrm{OH})_{2}\right)$, $7.97\left(\mathrm{dd}, J 2.8\right.$ and $\left.1.2 \mathrm{~Hz}, 1 \mathrm{H}, \mathrm{H}_{\text {ary }}\right), 7.46(\mathrm{dd}, J 4.4$ and $\left.2.4 \mathrm{~Hz}, 1 \mathrm{H}, \mathrm{H}_{\text {ary }}\right), 7.42\left(\mathrm{dd}, J 4.4\right.$ and $\left.0.8 \mathrm{~Hz}, 1 \mathrm{H}, \mathrm{H}_{\text {ary }}\right)$; ${ }^{13} \mathrm{C}$ NMR(100 MHz, DMSO- $\left.d_{6}\right) \delta 134.9,132.5,125.1$; ${ }^{11} \mathrm{~B}$ NMR (128 MHz, DMSO- $\left.d_{6}\right) \delta 26.7$ (s). This is in agreement with data previously reported. ${ }^{14}$

\section{Dibenzo[b, $d]$ furan-2-ylboronic acid (2n)}

Yield: $91 \mathrm{mg}$ (86\%); white solid; $\mathrm{mp} 170-172{ }^{\circ} \mathrm{C}$; ${ }^{1} \mathrm{H}$ NMR (400 MHz, DMSO- $\left.d_{6}\right) \delta 8.28\left(\mathrm{~s}, 2 \mathrm{H}, \mathrm{B}(\mathrm{OH})_{2}\right)$, $8.17\left(\mathrm{dd}, J 7.2\right.$ and $\left.1.2 \mathrm{~Hz}, 1 \mathrm{H}, \mathrm{H}_{\text {ary }}\right), 8.12(\mathrm{dd}, J 7.2$ and $\left.0.8 \mathrm{~Hz}, 1 \mathrm{H}, \mathrm{H}_{\text {ary }}\right), 7.75\left(\mathrm{dd}, J 7.6\right.$ and $\left.1.6 \mathrm{~Hz}, 1 \mathrm{H}, \mathrm{H}_{\text {ary }}\right), 7.71$ (dd, $J 8.0$ and $\left.0.8 \mathrm{~Hz}, 1 \mathrm{H}, \mathrm{H}_{\text {ary }}\right), 7.53-7.49\left(\mathrm{~m}, 1 \mathrm{H}, \mathrm{H}_{\text {ary }}\right)$, 7.40-7.36 (m, $\left.2 \mathrm{H}, \mathrm{H}_{\text {ary }}\right) ;{ }^{13} \mathrm{C}$ NMR (100 MHz, DMSO- $\left.d_{6}\right)$ $\delta$ 159.2, 155.3, 133.1, 127.3, 123.5, 122.8, 122.6, 122.5, 122.4, 120.9, 111.6; ${ }^{11} \mathrm{~B}$ NMR (128 MHz, DMSO- $\left.d_{6}\right) \delta 28.9$ (s). This is in agreement with data previously reported. ${ }^{21}$
Phenethylboronic acid (20)

Yield: $64.5 \mathrm{mg}(86 \%)$; white solid; $\mathrm{mp} 59-61{ }^{\circ} \mathrm{C}$; ${ }^{1} \mathrm{H}$ NMR (400 MHz, DMSO- $\left.d_{6}\right) \delta 7.51\left(\mathrm{~s}, 2 \mathrm{H}, \mathrm{B}(\mathrm{OH})_{2}\right)$, 7.24 (dd, $J 7.6$ and $\left.7.2 \mathrm{~Hz}, 2 \mathrm{H}, \mathrm{H}_{\text {ary }}\right), 7.18(\mathrm{~d}, J 6.8 \mathrm{~Hz}, 2 \mathrm{H}$, $\left.\mathrm{H}_{\text {ary }}\right), 7.12\left(\mathrm{t}, J 7.6\right.$ and $\left.7.2 \mathrm{~Hz}, 1 \mathrm{H}, \mathrm{H}_{\text {aryl }}\right), 2.64(\mathrm{t}, J 8.4 \mathrm{~Hz}$, $\left.2 \mathrm{H}, \mathrm{CH}_{2}\right), 0.92\left(\mathrm{t}, J 8.4 \mathrm{~Hz}, 2 \mathrm{H}, \mathrm{CH}_{2}\right) ;{ }^{13} \mathrm{C} \mathrm{NMR}(100 \mathrm{MHz}$, DMSO- $\left.d_{6}\right) \delta 145.1,128.1,127.7,125.2,30.2 ;{ }^{11} \mathrm{~B}$ NMR $\left(128 \mathrm{MHz}, \mathrm{DMSO}-d_{6}\right) \delta 30.4$ (s). This is in agreement with data previously reported. ${ }^{14}$

General procedure for the synthesis of boronic esters from potassium organotrifluoroborates (3a-3m)

In a flask containing the appropriate boronic acid 1a-m (0.25 mmol) in tetrahydrofuran (THF; $3 \mathrm{~mL}$ ) was added montmorillonite K10 $(150 \% \mathrm{~m} / \mathrm{m})$ followed by pinacol (30 mg, $0.25 \mathrm{mmol}$ ). The mixture was stirred for $15 \mathrm{~min}$ to homogenize the contents, and powdered $4 \AA$ molecular sieves $(150 \% \mathrm{~m} / \mathrm{m})$ were added. The mixture was then stirred at room temperature for the time indicated in Scheme 2. After this period, the mixture was extracted with EtOAc $(3 \times 10 \mathrm{~mL})$ and the organic phase was washed with water $(2 \times 15 \mathrm{~mL})$. The organic phase was dried over anhydrous $\mathrm{MgSO}_{4}$, filtered and the solvent was removed in vacuo.

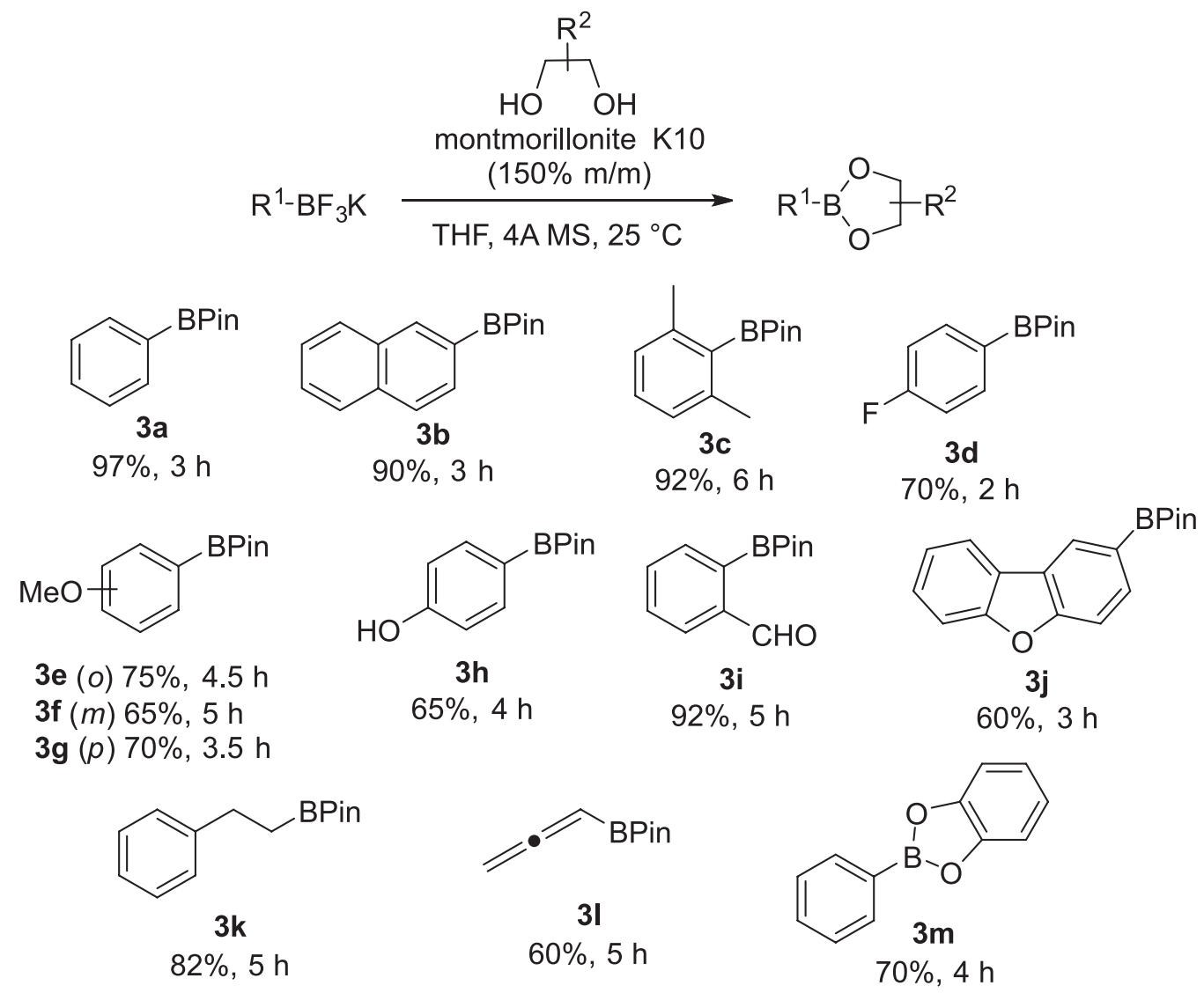

Scheme 2. Synthesis of different boronic esters from potassium organotrifluoroborates and alcohols using montmorillonite K10. 
4,4,5,5-Tetramethyl-2-phenyl-1,3,2-dioxaborolane (3a)

Yield: $50 \mathrm{mg}$ (97\%); oil; ${ }^{1} \mathrm{H}$ NMR (400 MHz, $\mathrm{CDCl}_{3}$ ) $\delta 7.83\left(\mathrm{~d}, J 7.2 \mathrm{~Hz}, 2 \mathrm{H}, \mathrm{H}_{\text {aryl }}\right), 7.47\left(\mathrm{t}, J 7.2 \mathrm{~Hz}, 1 \mathrm{H}, \mathrm{H}_{\text {aryl }}\right)$, 7.38 (dd, $J 7.2$ and $\left.6.8 \mathrm{~Hz}, 2 \mathrm{H}, \mathrm{H}_{\text {aryl }}\right), 1.36\left(\mathrm{~s}, 12 \mathrm{H}, \mathrm{CH}_{3}\right)$; ${ }^{13} \mathrm{C}$ NMR (100 MHz, $\left.\mathrm{CDCl}_{3}\right) \delta 134.7,131.2,127.7,83.8$, 24.9; ${ }^{11} \mathrm{~B}$ NMR $\left(128 \mathrm{MHz}, \mathrm{CDCl}_{3}\right) \delta 30.9$ (s). This is in agreement with data previously reported. ${ }^{18}$

4,4,5,5-Tetramethyl-2-(naphthalen-2-yl)-1,3,2-dioxaborolane (3b)

Yield: $57 \mathrm{mg}$ (90\%); waxy solid; ${ }^{1} \mathrm{H}$ NMR (400 MHz, $\left.\mathrm{CDCl}_{3}\right) \delta 8.79\left(\mathrm{dd}, J 8.8\right.$ and $\left.0.8 \mathrm{~Hz}, 1 \mathrm{H}, \mathrm{H}_{\text {aryl }}\right), 8.11$ (dd, $J 7.2$ and $\left.1.6 \mathrm{~Hz}, 1 \mathrm{H}, \mathrm{H}_{\text {aryl }}\right), 7.95\left(\mathrm{~d}, J 8.4 \mathrm{~Hz}, 1 \mathrm{H}, \mathrm{H}_{\text {aryl }}\right.$ ), $7.86\left(\mathrm{dd}, J 8.0\right.$ and $\left.0.8 \mathrm{~Hz}, 1 \mathrm{H}, \mathrm{H}_{\text {aryl }}\right), 7.58-7.54(\mathrm{~m}, 1 \mathrm{H}$, $\left.\mathrm{H}_{\text {aryl }}\right), 7.51-7.47\left(\mathrm{~m}, 2 \mathrm{H}, \mathrm{H}_{\text {aryl }}\right), 1.45\left(\mathrm{~s}, 12 \mathrm{H}, \mathrm{CH}_{3}\right) ;{ }^{13} \mathrm{C} \mathrm{NMR}$ $\left(100 \mathrm{MHz} \mathrm{CDCl}_{3}\right) \delta 136.9,135.6,133.2,131.6,128.4$, 128.3, 126.3, 125.4, 124.9, 83.7, 24.9; ${ }^{11} \mathrm{~B}$ NMR (128 MHz, $\left.\mathrm{CDCl}_{3}\right) \delta 31.4(\mathrm{~s})$. This is in agreement with data previously reported. ${ }^{22}$

2-(2,6-Dimethylphenyl)-4,4,5,5-tetramethyl-1,3,2-dioxaborolane (3c)

Yield: $53.5 \mathrm{mg}$ (92\%); oil; ${ }^{1} \mathrm{H}$ NMR (400 MHz, $\left.\mathrm{CDCl}_{3}\right)$ $\delta 6.98\left(\mathrm{t}, J 7.6 \mathrm{~Hz}, 1 \mathrm{H}, \mathrm{H}_{\text {aryl }}\right), 6.81\left(\mathrm{~d}, J 7.6 \mathrm{~Hz}, 2 \mathrm{H}, \mathrm{H}_{\text {aryl }}\right)$, $2.28\left(\mathrm{~s}, 6 \mathrm{H}, \mathrm{CH}_{3}\right), 1.22\left(\mathrm{~s}, 12 \mathrm{H}, \mathrm{CH}_{3}\right) ;{ }^{13} \mathrm{C} \mathrm{NMR}(100 \mathrm{MHz}$, $\left.\mathrm{CDCl}_{3}\right) \delta 141.5,128.9,126.2,83.3,24.7,22.0 ;{ }^{11} \mathrm{~B} \mathrm{NMR}$ $\left(128 \mathrm{MHz} \mathrm{CDCl}_{3}\right) \delta 32.3(\mathrm{~s})$. This is in agreement with data previously reported. ${ }^{23}$

\section{2-(4-Fluorophenyl)-4,4,5,5-tetramethyl-1,3,2-dioxaborolane} (3d)

Yield: $39 \mathrm{mg}$ (70\%); white solid; mp 51-52 ${ }^{\circ} \mathrm{C} ;{ }^{1} \mathrm{H}$ NMR $\left(400 \mathrm{MHz} \mathrm{CDCl}_{3}\right) \delta 7.82\left(\mathrm{t}, J 7.2 \mathrm{~Hz}, 2 \mathrm{H}, \mathrm{H}_{\text {aryl }}\right), 7.06$ (dd, $J 8.8$ and $\left.8.0 \mathrm{~Hz}, 2 \mathrm{H}, \mathrm{H}_{\text {aryl }}\right), 1.35\left(\mathrm{~s}, 12 \mathrm{H}, \mathrm{CH}_{3}\right) ;{ }^{13} \mathrm{C} \mathrm{NMR}$ $\left(100 \mathrm{MHz}, \mathrm{CDCl}_{3}\right) \delta 165.1\left(\mathrm{~d}, J_{\mathrm{C}-\mathrm{F}} 248.9 \mathrm{~Hz}, \mathrm{C}_{\text {aryl }}\right), 136.9$ $\left(\mathrm{d}, J_{\mathrm{C}-\mathrm{F}} 8.4 \mathrm{~Hz}, \mathrm{C}_{\text {aryl }}\right), 114.7$ (d, $\left.J_{\mathrm{C}-\mathrm{F}} 20.5 \mathrm{~Hz}, \mathrm{C}_{\text {aryl }}\right), 83.8$, $24.8 ;{ }^{11} \mathrm{~B}$ NMR $\left(128 \mathrm{MHz}, \mathrm{CDCl}_{3}\right) \delta 30.6(\mathrm{~s}) ;{ }^{19} \mathrm{~F} \mathrm{NMR}$ $\left(376 \mathrm{MHz}, \mathrm{CDCl}_{3}\right) \delta-108.4$ (s). This is in agreement with data previously reported. ${ }^{18}$

2-(2-Methoxyphenyl)-4,4,5,5-tetramethyl-1,3,2-dioxaborolane (3e)

Yield: 44 mg (75\%); oil; ${ }^{1} \mathrm{H}$ NMR (400 MHz, $\mathrm{CDCl}_{3}$ ) $\delta 7.41\left(\mathrm{~d}, J 7.6 \mathrm{~Hz}, 1 \mathrm{H}, \mathrm{H}_{\text {aryl }}\right), 7.34(\mathrm{~d}, J 2.8 \mathrm{~Hz}, 1 \mathrm{H}$, $\left.\mathrm{H}_{\text {aryl }}\right), 7.32-7.26\left(\mathrm{~m}, 1 \mathrm{H}, \mathrm{H}_{\text {aryl }}\right), 7.02$ (ddd, J 8.0, 2.8 and $\left.0.8 \mathrm{~Hz}, 1 \mathrm{H}, \mathrm{H}_{\text {aryl }}\right), 3.84$ (s, 3H, $\left.\mathrm{OCH}_{3}\right), 1.35\left(\mathrm{~s}, 12 \mathrm{H}, \mathrm{CH}_{3}\right)$; ${ }^{13} \mathrm{C} \mathrm{NMR}\left(100 \mathrm{MHz}, \mathrm{CDCl}_{3}\right) \delta 158.9,128.8,127.1,118.7$, 117.7, 83.7, 55.1, 24.8; ${ }^{11} \mathrm{~B}$ NMR (128 $\left.\mathrm{MHz} \mathrm{CDCl}_{3}\right)$ $\delta 28.2$ (s). This is in agreement with data previously reported. ${ }^{24}$
2-(3-Methoxyphenyl)-4,4,5,5-tetramethyl-1,3,2-dioxaborolane (3f)

Yield: $38 \mathrm{mg}$ (65\%); oil; ${ }^{1} \mathrm{H} \mathrm{NMR}\left(400 \mathrm{MHz}, \mathrm{CDCl}_{3}\right.$ ) $\delta 7.72\left(\mathrm{dd}, J 7.2\right.$ and $\left.1.6 \mathrm{~Hz}, 1 \mathrm{H}, \mathrm{H}_{\text {aryl }}\right), 7.43-7.39(\mathrm{~m}$, $\left.1 \mathrm{H}, \mathrm{H}_{\text {aryl }}\right), 6.96\left(\mathrm{dd}, J 7.6\right.$ and $\left.6.8 \mathrm{~Hz}, 1 \mathrm{H}, \mathrm{H}_{\text {aryl }}\right), 6.87$ (d, $\left.J 8.4 \mathrm{~Hz}, 1 \mathrm{H}, \mathrm{H}_{\text {aryl }}\right), 3.84\left(\mathrm{~s}, 3 \mathrm{H}, \mathrm{OCH}_{3}\right), 1.38\left(\mathrm{~s}, 12 \mathrm{H}, \mathrm{CH}_{3}\right)$; ${ }^{13} \mathrm{C} \mathrm{NMR}\left(100 \mathrm{MHz}, \mathrm{CDCl}_{3}\right) \delta 164.0,136.6,132.3,120.0$, 110.3, 83.3, 55.6, 24.7; ${ }^{11} \mathrm{~B}$ NMR (128 MHz, $\left.\mathrm{CDCl}_{3}\right) \delta 28.4$ (s). This is in agreement with data previously reported. ${ }^{24}$

2-(4-Methoxyphenyl)-4,4,5,5-tetramethyl-1,3,2-dioxaborolane $(\mathbf{3 g})$

Yield: $41 \mathrm{mg}$ (70\%); oil; ${ }^{1} \mathrm{H}$ NMR (400 MHz, $\mathrm{CDCl}_{3}$ ) $\delta 7.78\left(\mathrm{~d}, J 8.8 \mathrm{~Hz}, 2 \mathrm{H}, \mathrm{H}_{\text {aryl }}\right), 6.91(\mathrm{~d}, J 8.4 \mathrm{~Hz}, 2 \mathrm{H}$, $\left.\mathrm{H}_{\text {aryl }}\right), 3.83\left(\mathrm{~s}, 3 \mathrm{H}, \mathrm{OCH}_{3}\right), 1.35\left(\mathrm{~s}, 12 \mathrm{H}, \mathrm{CH}_{3}\right) ;{ }^{13} \mathrm{C} \mathrm{NMR}$ $\left(100 \mathrm{MHz}, \mathrm{CDCl}_{3}\right) \delta 162.1,136.4,113.2,83.4,54.9,24.8$; ${ }^{11} \mathrm{~B}$ NMR $\left(128 \mathrm{MHz}, \mathrm{CDCl}_{3}\right) \delta 28.1$ (s). This is in agreement with data previously reported. ${ }^{24}$

4-(4,4,5,5-Tetramethyl-1,3,2-dioxaborolan-2-yl)phenol (3h) Yield: $36 \mathrm{mg}$ (65\%); white solid; mp 114-116 ${ }^{\circ} \mathrm{C}$; ${ }^{1} \mathrm{H} \mathrm{NMR}\left(400 \mathrm{MHz}, \mathrm{CDCl}_{3}\right) \delta 7.71\left(\mathrm{~d}, J 8.4 \mathrm{~Hz}, 2 \mathrm{H}, \mathrm{H}_{\text {aryl }}\right)$, $6.83\left(\mathrm{~d}, J 8.4 \mathrm{~Hz}, 2 \mathrm{H}, \mathrm{H}_{\text {aryl }}\right), 6.32$ (s, $\left.1 \mathrm{H}, \mathrm{OH}\right), 1.35$ (s, 12H, $\left.\mathrm{CH}_{3}\right) ;{ }^{13} \mathrm{C} \mathrm{NMR}\left(100 \mathrm{MHz}, \mathrm{CDCl}_{3}\right) \delta 158.6,136.7,114.9$, 83.8, 24.7; ${ }^{11} \mathrm{~B}$ NMR (128 MHz, $\left.\mathrm{CDCl}_{3}\right) \delta 28.2$ (s). This is in agreement with data previously reported. ${ }^{25}$

2-(4,4,5,5-Tetramethyl-1,3,2-dioxaborolan-2-yl) benzaldehyde (3i)

Yield: 53.5 mg (92\%); oil; ${ }^{1} \mathrm{H} \mathrm{NMR}$ (400 MHz, $\mathrm{CDCl}_{3}$ ) $\delta 10.55(\mathrm{~s}, 1 \mathrm{H}, \mathrm{CHO}), 7.96(\mathrm{dd}, J 7.2$ and $1.2 \mathrm{~Hz}, 1 \mathrm{H}$, $\left.\mathrm{H}_{\text {aryl }}\right), 7.86\left(\mathrm{dd}, J 7.2\right.$ and $\left.1.6 \mathrm{~Hz}, 1 \mathrm{H}, \mathrm{H}_{\text {aryl }}\right), 7.61-7.54$ $\left(\mathrm{m}, 2 \mathrm{H}, \mathrm{H}_{\text {aryl }}\right), 1.40\left(\mathrm{~s}, 12 \mathrm{H}, \mathrm{CH}_{3}\right) ;{ }^{13} \mathrm{C} \mathrm{NMR}(100 \mathrm{MHz}$, $\left.\mathrm{CDCl}_{3}\right) \delta 194.6,141.2,135.4,132.9,130.7,127.8,84.3$, 24.8; ${ }^{11} \mathrm{~B} \mathrm{NMR}\left(128 \mathrm{MHz}, \mathrm{CDCl}_{3}\right) \delta 31.2$ (s). This is in agreement with data previously reported. ${ }^{26}$

2-(Dibenzo[ $b, d]$ furan-2-yl)-4,4,5,5-tetramethyl-1,3,2-dioxaborolane (3j)

Yield: $44 \mathrm{mg}(60 \%)$; white solid; mp 75-76 ${ }^{\circ} \mathrm{C}$; ${ }^{1} \mathrm{H} \mathrm{NMR}\left(400 \mathrm{MHz}, \mathrm{CDCl}_{3}\right) \delta 7.96(\mathrm{dd}, J 8.0$ and $1.6 \mathrm{~Hz}$, $\left.1 \mathrm{H}, \mathrm{H}_{\text {aryl }}\right), 7.85-7.80\left(\mathrm{~m}, 2 \mathrm{H}, \mathrm{H}_{\text {aryl }}\right), 7.58(\mathrm{~d}, J 8.4 \mathrm{~Hz}, 1 \mathrm{H}$, $\left.\mathrm{H}_{\text {aryl }}\right), 7.36-7.32\left(\mathrm{~m}, 1 \mathrm{H}, \mathrm{H}_{\text {aryl }}\right), 7.26(\mathrm{dd}, J 7.6$ and $7.2 \mathrm{~Hz}$, $\left.1 \mathrm{H}, \mathrm{H}_{\text {aryl }}\right), 7.21\left(\mathrm{~d}, J 7.2 \mathrm{~Hz}, 1 \mathrm{H}, \mathrm{H}_{\text {aryl }}\right), 1.35\left(\mathrm{~s}, 12 \mathrm{H}, \mathrm{CH}_{3}\right)$; ${ }^{13} \mathrm{C}$ NMR $\left(100 \mathrm{MHz}, \mathrm{CDCl}_{3}\right) \delta 160.4,156.3,134.4,126.9$, 123.9, 123.6, 123.2, 122.4, 122.2, 120.3, 112.2, 84.0, 24.8; ${ }^{11} \mathrm{~B}$ NMR $\left(128 \mathrm{MHz}, \mathrm{CDCl}_{3}\right) \delta 30.6(\mathrm{~s})$. This is in agreement with data previously reported. ${ }^{27}$

4,4,5,5-Tetramethyl-2-phenethyl-1,3,2-dioxaborolane (3k) Yield: $47.5 \mathrm{mg}$ (82\%); oil; ${ }^{1} \mathrm{H}$ NMR (400 MHz, $\mathrm{CDCl}_{3}$ ) 
$\delta$ 7.19-7.05 (m, 4H, $\left.\mathrm{H}_{\text {ary }}\right), 7.09-7.04\left(\mathrm{~m}, 1 \mathrm{H}, \mathrm{H}_{\text {aryl }}\right), 2.67$ (t, $\left.J 8.0 \mathrm{~Hz}, 2 \mathrm{H}, \mathrm{CH}_{2}\right), 1.14\left(\mathrm{~s}, 12 \mathrm{H}, \mathrm{CH}_{3}\right), 1.07(\mathrm{t}, J 8.0 \mathrm{~Hz}, 2 \mathrm{H}$, $\left.\mathrm{CH}_{2}\right) ;{ }^{13} \mathrm{C}$ NMR $\left(100 \mathrm{MHz}, \mathrm{CDCl}_{3}\right) \delta 144.4,128.1,127.9$, 125.4, 83.1, 29.9, 24.8; ${ }^{11} \mathrm{~B} \mathrm{NMR}\left(128 \mathrm{MHz}, \mathrm{CDCl}_{3}\right) \delta 33.8$ (s). This is in agreement with data previously reported. ${ }^{28}$

\section{4,4,5,5-Tetramethyl-2-(propa-1,2-dien-1-yl)-1,3,2-dioxa- borolane (3I)}

Yield: $25 \mathrm{mg}$ (60\%); oil; ${ }^{1} \mathrm{H}$ NMR (400 MHz, $\mathrm{CDCl}_{3}$ ) $\delta 4.92(\mathrm{t}, J 6.8 \mathrm{~Hz}, 1 \mathrm{H}, \mathrm{CH}), 4.65\left(\mathrm{~d}, J 6.8 \mathrm{~Hz}, 2 \mathrm{H}, \mathrm{CH}_{2}\right)$, $1.28\left(\mathrm{~s}, 12 \mathrm{H}, \mathrm{CH}_{3}\right) ;{ }^{13} \mathrm{C} \mathrm{NMR}\left(100 \mathrm{MHz}, \mathrm{CDCl}_{3}\right) \delta 218.9$, 83.7, 70.0, 24.7; ${ }^{11} \mathrm{~B}$ NMR (128 MHz, $\left.\mathrm{CDCl}_{3}\right) \delta 29.4$ (s). This is in agreement with data previously reported. ${ }^{29}$

\section{2-Phenylbenzo[ $d][1,3,2]$ dioxaborole (3m)}

Yield: $34.5 \mathrm{mg}$ (70\%); oil; ${ }^{1} \mathrm{H} \mathrm{NMR}\left(400 \mathrm{MHz}, \mathrm{CDCl}_{3}\right)$ $\delta 8.11\left(\mathrm{dd}, J 8.0\right.$ and $\left.1.2 \mathrm{~Hz}, 2 \mathrm{H}, \mathrm{H}_{\text {aryl }}\right), 7.62-7.57(\mathrm{~m}, 1 \mathrm{H}$, $\left.\mathrm{H}_{\text {ary }}\right), 7.53-7.48\left(\mathrm{~m}, 2 \mathrm{H}, \mathrm{H}_{\text {ary }}\right), 7.35-7.13\left(\mathrm{~m}, 2 \mathrm{H}, \mathrm{H}_{\text {aryl }}\right)$, 7.16-7.13 (m, 2H, $\left.\mathrm{H}_{\text {ary }}\right) ;{ }^{13} \mathrm{C}$ NMR $\left(100 \mathrm{MHz}, \mathrm{CDCl}_{3}\right)$ $\delta 148.5,134.9,132.3,128.2,122.7,112.5 ;{ }^{11} \mathrm{~B}$ NMR $\left(128 \mathrm{MHz}, \mathrm{CDCl}_{3}\right) \delta 28.3(\mathrm{~s})$. This is in agreement with data previously reported. ${ }^{30}$

\section{Results and Discussion}

For preliminary optimization of the reaction conditions, a solution of potassium aryltrifluoroborate $1 \mathbf{a}(1 \mathrm{mmol})$ in $\mathrm{H}_{2} \mathrm{O}$ was treated with different clays at room temperature. The results are depicted in Table 1.

From Table 1, it can be seen that when montmorillonites were used, the conversion of $\mathbf{1 a}$ to the corresponding boronic acid 2a was quantitative (Table 1, entries 2 and

Table 1. Influence of different amount of montmorillonite K10 in the hydrolysis of $\mathbf{1 a}$ at $25^{\circ} \mathrm{C}^{\text {a }}$

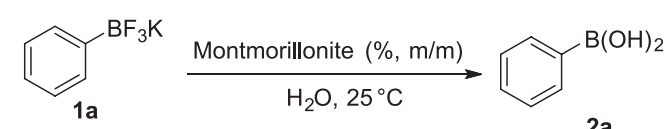

\begin{tabular}{lcccc}
\hline entry & Montmorillonite & $\begin{array}{c}\text { Amount } / \\
\%(\mathrm{~m} / \mathrm{m})\end{array}$ & time / min & $\mathbf{2 a}^{\mathrm{b}} / \%$ \\
\hline 1 & - & - & 90 & 0 \\
2 & KSF & 100 & 60 & 100 \\
3 & K10 & 100 & 40 & 100 \\
4 & K10 & 200 & 25 & 100 \\
5 & K10 & 150 & 30 & 100 \\
6 & K10 & 50 & 90 & 100 \\
7 & K10 & 25 & 90 & 60 \\
\hline
\end{tabular}

aReaction conditions: reactions were performed using $\mathbf{1 a}(0.5 \mathrm{mmol})$ in $\mathrm{H}_{2} \mathrm{O}(1.0 \mathrm{~mL})$ and different amounts of the appropriate montmorillonite

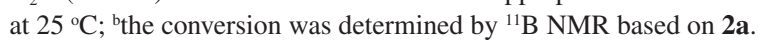

3). However, no product was observed when only $\mathrm{H}_{2} \mathrm{O}$ was used after $90 \mathrm{~min}$, indicating that the presence of additives is essential for the progress of the reaction. This result is probably due to the acidic character of the montmorillonites used and, mainly, due to the presence of the silicon atom in their structures. ${ }^{31}$ Since silicon presents a strong interaction with fluorine atom, this would favor the cleavage of the B-F bond in the tetrahedral structure of compound 1a, leading to the formation of the intermediate phenyldifluoroborane, which immediately undergoes a nucleophilic attack from water. Lennox and Lloyd-Jones ${ }^{32}$ detailed the mechanism of hydrolysis as an equilibrium between the boronic acid and the organotrifluoroborate driven toward boronic acid due to the abstraction of fluoride, which can be promoted by a base and/or glassware. The differences in reaction times using the montmorillonite KSF or K10 can be explained by their surface areas. For montmorillonite clays the surface area of $\mathrm{K} 10$ has a higher value (about 220-270 $\mathrm{m}^{2} \mathrm{~g}^{-1}$ ) when compared to KSF $\left(20-40 \mathrm{~m}^{2} \mathrm{~g}^{-1}\right) .^{31}$

After that, the amount of montmorillonite K10 to promote the reaction was investigated. The amount of clay was changed from 200 to $25 \% \mathrm{~m} / \mathrm{m}$. It was observed that the conversion of $\mathbf{1 a}$ to $\mathbf{2 a}$ did not change considerably when the amount of the catalyst varied from 200 to $50 \% \mathrm{~m} / \mathrm{m}$ (Table 1, entries 4-6), however, when the amount of the catalyst was decreased to $25 \% \mathrm{~m} / \mathrm{m}$, a lower conversion was observed (Table 1, entry 7). Despite having similar conversions, the increase in the amount of the clay led to a considerable decrease in reaction time.

Finally, the most suitable solvent for the hydrolysis of 1a was investigated. As potassium aryltrifluoroborates are salts, it was expected that water would be the most appropriate solvent to perform the reaction. Accordingly, better results were achieved using water as the solvent, leading to the desired product quantitatively after $30 \mathrm{~min}$ reaction at room temperature (Table 2, entry 1). Although, when the same reaction was performed under reflux, a decrease in the conversion was observed (Table 2, entry 2). The use of a less polar solvent, such as THF, provided the corresponding boronic acid $\mathbf{2 a}$ also in good conversions (Table 2, entry 3). However, when dichloromethane was used, only $8 \%$ conversion was observed (Table 2, entry 4). Finally, when more polar solvents, such as methanol or acetonitrile were used in the reaction, lower conversions were observed (Table 2, entries 5-6).

The optimized reaction conditions, namely $\mathbf{1 a}$ $(0.5 \mathrm{mmol})$, montmorillonite $\mathrm{K} 10(150 \% \mathrm{~m} / \mathrm{m})$ in $\mathrm{H}_{2} \mathrm{O}$ $(1.0 \mathrm{~mL})$ at $25^{\circ} \mathrm{C}$, were then applied for the hydrolysis of several potassium aryltrifluoroborates containing a wide range of functional groups and the results are shown in Scheme 1. 
Table 2. Influence of different solvents on the hydrolysis of $\mathbf{1 a}$ at $25{ }^{\circ} \mathrm{C}$ using montmorillonite $\mathrm{K} 10^{\mathrm{a}}$

\begin{tabular}{|c|c|c|}
\hline & 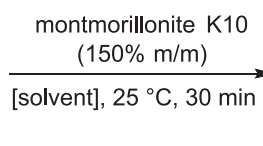 & $-\mathrm{B}(\mathrm{OH})_{2}$ \\
\hline entry & Solvent & Yield $/ \%$ \\
\hline 1 & $\mathrm{H}_{2} \mathrm{O}$ & 100 \\
\hline 2 & $\mathrm{H}_{2} \mathrm{O}$ & $60^{c}$ \\
\hline 3 & THF & 83 \\
\hline 4 & $\mathrm{CH}_{2} \mathrm{Cl}_{2}$ & 8 \\
\hline 5 & $\mathrm{MeCN}$ & 26 \\
\hline 6 & $\mathrm{MeOH}$ & 48 \\
\hline
\end{tabular}

Reaction conditions: reactions were performed using $\mathbf{1 a}(0.5 \mathrm{mmol})$ in the appropriate solvent $(1.0 \mathrm{~mL})$ and montmorillonite $\mathrm{K} 10(150 \% \mathrm{~m} / \mathrm{m})$

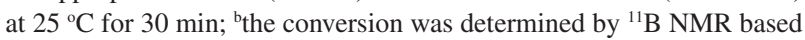
on $\mathbf{2} \mathbf{a}$; ${ }^{c}$ the reaction was conducted under reflux. THF: tetrahydrofuran.

From the results shown in Scheme 1, electron-donating or electron-withdrawing groups, as well as their position on the aromatic ring, have a dramatic influence on the product yield and reaction time. This fact is in accordance with previous work described by Perrin and co-workers ${ }^{15}$ where the rate of organotrifluoroborate solvolysis is governed by substituent groups. ${ }^{32}$ For example, when potassium 2-, 3- or 4-methoxyphenyltrifluoroborates were used, the corresponding products $\mathbf{2} \mathbf{b}-\mathbf{d}$ were obtained in good yields and in different reaction times, being the 4-derivative obtained in higher yield and shorter reaction time. Potassium aryltrifluoroborates containing hydroxyl or amino groups were also efficiently hydrolyzed. This is an interesting result as it was previously described that the hydrolysis of 2e using lithium hydroxide resulted in decomposition of the starting material to a dark polymeric mixture. ${ }^{33}$ When potassium organotrifluoroborates containing halogens were used, the corresponding products $\mathbf{2 g}$ and $\mathbf{2 h}$ were also obtained in good yields.

Next, attention was turned to electron-poor aryltrifluoroborates, which as expected, required a longer time for complete deprotection. As an example, potassium organotrifluoroborate containing an aldehyde $\mathbf{2} \mathbf{i}$ was obtained in moderate yield after $24 \mathrm{~h}$. The same behavior was observed for compound $\mathbf{2 l}$, obtained in $90 \%$ after $24 \mathrm{~h}$.

Sterically-hindered potassium aryltrifluoroborates provided the corresponding arylboronic acids $\mathbf{2} \mathbf{j}$ and $\mathbf{2 k}$ in good yields and short reaction time. The use of potassium heteroaryltrifluoroborates led to the corresponding boronic acids $\mathbf{2 m}$ and $\mathbf{2 n}$ in excellent yields.

Finally, the hydrolysis of non-aromatic trifluoroborates was examined, and the conditions developed were proven to be effective, giving 20 in good yield after $1 \mathrm{~h}$. However, the reaction was not efficient for the allenyl derivative, where $2 \mathbf{p}$ was not obtained and only a complex mixture of compounds was observed.

In addition, the use of montmorillonite K10 was applied in the synthesis of some boronic esters $\mathbf{3}$, which present higher stability comparing with boronic acids 2 .

In a first attempt, the same reaction conditions for the hydrolysis of potassium organotrifluoroborates were used, however, equimolar amounts of pinacol were added to the reaction. The reaction was monitored by ${ }^{11} \mathrm{~B} \mathrm{NMR}$ and after $1 \mathrm{~h}$, a mixture of the desired compound $\mathbf{3 a}$, boronic acid $\mathbf{2 a}$ and the starting material was observed. When the reaction was carried out employing a longer reaction time, the same result was observed (Table 3, entries 1 and 2).

Table 3. Influence of different solvents on the synthesis of $\mathbf{3 a}$ at $25{ }^{\circ} \mathrm{C}$ using montmorillonite $\mathrm{K} 10^{\mathrm{a}}$

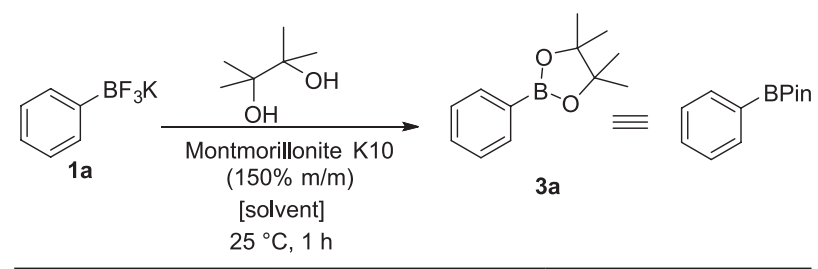

\begin{tabular}{lcc}
\hline entry & Solvent & $\mathbf{3 a}^{\mathrm{b}} / \%$ \\
\hline 1 & $\mathrm{H}_{2} \mathrm{O}$ & 30 \\
2 & $\mathrm{H}_{2} \mathrm{O}$ & $30^{\mathrm{c}}$ \\
3 & $\mathrm{EtOAc}$ & 40 \\
4 & DMF & 25 \\
5 & dioxane & 52 \\
6 & MeCN & 62 \\
7 & THF & 70 \\
8 & THF & $100^{\mathrm{d}}$ \\
\hline
\end{tabular}

${ }^{\mathrm{a}}$ Reaction conditions: reactions were performed using 1a $(0.25 \mathrm{mmol})$ and pinacol $(0.25 \mathrm{mmol})$ in the appropriate solvent $(1.0 \mathrm{~mL})$ and montmorillonite $\mathrm{K} 10(150 \% \mathrm{~m} / \mathrm{m})$ at $25{ }^{\circ} \mathrm{C}$ for $1 \mathrm{~h}$; ${ }^{b}$ the conversion was determined by ${ }^{11} \mathrm{~B}$ NMR based on $\mathbf{3 a}$; ${ }^{c}$ the reaction was stirred for $12 \mathrm{~h}$; ${ }^{\mathrm{d}}$ the reaction was performed using $4 \AA$ molecular sieves $(100 \% \mathrm{~m} / \mathrm{m})$ at $25^{\circ} \mathrm{C}$ for $3 \mathrm{~h}$. DMF: $N, N$-dimethylformamide; THF: tetrahydrofuran.

This result indicates that the presence of water in the reaction might be a problem for the formation of $\mathbf{3 a}$ and, in an attempt to improve the conversion to $\mathbf{3 a}$, different solvents were screened (Table 3).

Low conversions were observed when ethyl acetate, $N, N$-dimethylformamide or dioxane were used in the reaction (Table 3, entries 3-5). The use of acetonitrile also gave the product in lower conversions (Table 3, entry 6). From the different solvents employed to promote the reaction, the best result was observed when THF was used, where 3a was obtained in $70 \%$ conversion determined by ${ }^{11} \mathrm{~B}$ NMR after $1 \mathrm{~h}$ (Table 3, entry 7). It is interesting to 
note that the conversion was maintained even after $3 \mathrm{~h}$ of reaction. Of note, the addition of $4 \AA$ molecular sieves to the reaction medium led to the total consumption of pinacol after $3 \mathrm{~h}$ and $\mathbf{3 a}$ was obtained as the sole product in a $100 \%$ conversion (Table 3 , entry 8 ).

The robustness of the developed method was tested by applying the reaction conditions for the synthesis of other different boronic esters. The results are summarized in Scheme 2.

From Scheme 2, it can be seen that the presence of electron-donating or electron-withdrawing groups did not significantly interfere with the reaction. However, the reaction time presented variation depending on the substrate structure. For example, boronic ester $\mathbf{3 a}$ was obtained in $97 \%$ yield after $3 \mathrm{~h}$, and the reaction for $o$-disubstituted pinacol ester $3 \mathrm{c}$ required $6 \mathrm{~h}$ to be completed.

The synthesis of non-aromatic pinacol esters was also examined, and $\mathbf{3 k}$ was obtained in good yield after $5 \mathrm{~h}$. When potassium allenyl trifluoroborate was used, the corresponding boronic ester $3 \mathrm{l}$ was obtained in $60 \%$ yield after $5 \mathrm{~h}$. Finally, the use of catechol led to the corresponding boronic ester $\mathbf{3 m}$ in good yield after $4 \mathrm{~h}$, indicating that the method could be applied to other diols.

The recoverability and recyclability of the montmorillonite K10 was also evaluated after successive reactions using potassium phenyltrifluoroborate 1a. In the first cycles, the observed conversions to 2a were equivalent and a longer reaction time was required for the reaction completion. However, after the third cycle the observed conversions were lower. The results are summarized in Table 4.

Table 4. Recyclability of montmorillonite K10 after successive reactions ${ }^{\mathrm{a}}$

\begin{tabular}{|c|c|c|c|}
\hline \multirow[b]{2}{*}{ entry } & \multicolumn{2}{|c|}{$\begin{array}{c}\begin{array}{c}\text { Montmorillonite K10 } \\
(150 \% \mathrm{~m} / \mathrm{m})\end{array} \\
\mathrm{H}_{2} \mathrm{O}, 25^{\circ} \mathrm{C}\end{array}$} & $(\mathrm{OH})_{2}$ \\
\hline & Cycle & time / min & $\mathbf{2} \mathbf{a}^{\mathrm{b}} / \%$ \\
\hline 1 & - & 30 & 100 \\
\hline 2 & 1 & 60 & 95 \\
\hline 3 & 2 & 120 & 94 \\
\hline 4 & 3 & 120 & 60 \\
\hline 5 & 4 & 120 & 58 \\
\hline
\end{tabular}

Reaction conditions: reactions were performed using $1 \mathrm{a}(0.5 \mathrm{mmol})$ in water $(1.0 \mathrm{~mL})$ and montmorillonite $\mathrm{K} 10(150 \% \mathrm{~m} / \mathrm{m})$ at $25^{\circ} \mathrm{C}$ for the time indicated. At the end of the reaction, the supernatant was decanted and the remaining solid washed with water $(2 \times 1.0 \mathrm{~mL})$; ${ }^{\text {b the conversion }}$ was determined by ${ }^{11} \mathrm{~B}$ NMR based on $\mathbf{2 a}$.

\section{Conclusions}

In summary, an efficient and non-expensive method for conversion of diverse potassium organotrifluoroborates to their corresponding boronic acids promoted by montmorillonite K10 using water as the reaction solvent was described. Further interconversion of potassium organotrifluoroborates to their corresponding boronic esters, via boronic acid intermediates, was also successfully accomplished. The method is simple, fast and general, allowing further applications in the synthesis of more complex compounds.

\section{Supplementary Information}

Supplementary information (additional experimental procedures and spectroscopic characterization data, as well as ${ }^{1} \mathrm{H},{ }^{13} \mathrm{C},{ }^{11} \mathrm{~B}$ and ${ }^{19} \mathrm{~F}$ NMR spectra for all synthesized compounds) is available free of charge at http://jbcs.sbq.org.br as PDF file.

\section{Acknowledgments}

The authors gratefully acknowledge CNPq (482299/2013-4 and 447361/2014-7) and CAPES for financial support. P. H. M. and J. C. R. F. are also thankful to $\mathrm{CNPq}$ for their fellowship.

\section{References}

1. Hartwig, J. F.; Organotransition Metal Chemistry: From Bonding to Catalysis, $1^{\text {st }}$ ed.; University Science Books: Sausalito, 2010.

2. Sandrock, D. L.; Alkylboron Cross-Coupling Reactions. Science of Synthesis, Cross Coupling and Heck-Type Reactions 1; Georg Thieme Verlag: Stuttgart, 2013.

3. Nocentini, A.; Cadoni, R.; del Prete, S.; Capasso, C.; Dumy, P.; Gratteri, P.; Supuran, C. T.; Winum, J. Y.; ACS Med. Chem. Lett. 2017, 8, 1194; Winum, J.-Y.; Innocenti, A.; Scozzafava, A.; Montero, J.-L.; Supuran, C. T.; Bioorg. Med. Chem. 2009, 17, 3649 .

4. Roy, S.; Maiti, M.; Roy, A.; ChemistrySelect 2017, 2, 6929; Gao, X.; Zhang, Y.; Wang, B.; Org. Lett. 2003, 5, 4615; Coskun, A.; Akkaya, E. U.; Org. Lett. 2004, 6, 3107.

5. Jung, S. J.; Lee, J. Y.; Kim, T. H.; Lee, D. E.; Jeon, J.; Yang, S. D.; Hur, M. G.; Min, J. J.; Park, Y. D.; Bioorg. Med. Chem. 2016, 26, 1784; Kumar, S. K.; Hager, E.; Pettit, C.; Gurulingappa, H.; Davidson, N. E.; Khan, S. R.; J. Med. Chem. 2003, 46, 2813.

6. Miyaura, N.; Suzuki, A.; Chem. Rev. 1995, 95, 2457.

7. Hall, D. G.; Boronic Acids. Preparation, Applications in Organic Synthesis and Medicine, vol. 2, $2^{\text {nd }}$ ed.; Wiley-VCH: Weinheim, 2011.

8. Molander, G. A.; Canturk, B. C.; Angew. Chem., Int. Ed. 2009, 48, 9240 . 
9. Molander, G. A.; Sandrock, D. L.; Org. Lett. 2009, 11, 2369.

10. Woerly, E. M.; Roy, J.; Burke, M. D.; Nat. Chem. 2014, 6, 484; Fujii, S.; Chang, S. Y.; Burke, M. D.; Angew. Chem., Int. Ed. 2011, 50, 7862; Woerly, E. M.; Cherney, A. H.; Davis, E. K.; Burke, M. D.; J. Am. Chem. Soc. 2010, 132, 6941; Knapp, D. M.; Gillis, E. P.; Burke, M. D.; J. Am. Chem. Soc. 2009, 131, 6961; Uno, B. E.; Gillis, E. P.; Burke, M. D.; Tetrahedron 2009, 65, 3130; Gillis, E. P.; Burke, M. D.; Aldrichimica Acta 2009, 42, 17; Gillis, E. P.; Burke, M. D.; J. Am. Chem. Soc. 2008, 130, 14084; Lee, S. J.; Gray, K. C.; Paek, J. S.; Burke, M. D.; J. Am. Chem. Soc. 2008, 130, 466; Gillis, E. P.; Burke, M. D.; J. Am. Chem. Soc. 2007, 129, 6716.

11. Iwadate, N.; Suginome, M.; Org. Lett. 2009, 11, 1899; Noguchi, H.; Shioda, T.; Chou, C.-M.; Suginome, M.; Org. Lett. 2008, 10, 377; Noguchi, H.; Hojo, K.; Suginome, M.; J. Am. Chem. Soc. 2007, 129, 758.

12. Molander, G. A.; J. Org. Chem. 2015, 80, 7837.

13. Molander, G. A.; Gérard, L. J.; J. Org. Chem. 2007, 72, 8422; Lennox, A. J. J.; Jones, G. C. L.; Chem. Soc. Rev. 2014, 43, 412; Trost, B. M.; Science 1991, 254, 1471.

14. Churches, Q. I.; Hooper, J. F.; Hutton, C. A.; J. Org. Chem. 2015, 80, 5428; Blevins, D. W.; Yao, M. L.; Yong, L.; Kabalka, G. W.; Tetrahedron Lett. 2011, 52, 6534; Inglis, S. R.; Woon, E. C. Y.; Thompson, A. L.; Schofield, C. J.; J. Org. Chem. 2010, 75, 468; Kabalka, G. W.; Coltuclu, V.; Tetrahedron Lett. 2009, 50, 6271; Molander, G. A.; Cavalcanti, L. N.; Canturk, B.; Pan, P. S.; Kennedy, L. E.; J. Org. Chem. 2009, 74, 7364; Yuen, A. K. L.; Hutton, C. A.; Tetrahedron Lett. 2005, 46, 7899; Kim, B. J.; Matteson, D. S.; Angew. Chem., Int. Ed. 2004, 43, 3056; Pasumansky, L.; Haddenham, D.; Clary, J. W.; Fisher, G. B.; Goralski, C. T.; Sigaram, B.; J. Org. Chem. 2008, 73, 1898; Bruns, S.; Sinnwell, V.; Voss, J.; Magn. Reson. Chem. 2003, 41, 269; Kabalka, G. W.; Sastry, K. A. R.; Knapp Jr., F. F.; Srivastava, P. C.; J. Organomet. Chem. 1983, 259, 269; Cox, P. A.; Reid, M.; Leach, A. G.; Campbell, A. D.; King, E. J.; Lloyd-Jones, G. C.; J. Am. Chem. Soc. 2017, 139, 13156.

15. Ting, R.; Harwig, C. W.; Lo, J.; Li, Y.; Adam, M. J.; Ruth, T. J.; Perrin, D. M.; J. Org. Chem. 2008, 73, 4662.

16. Molander, G. A.; Sandrock, D. L.; J. Am. Chem. Soc. 2008, 130, 15792.
17. Perrin, D. D.; Armarego, W. L. F.; Purification of Laboratory Chemicals, $4^{\text {th }}$ ed.; Pergamon: Oxford, 1980.

18. Mfuh, A. M.; Doyle, J. D.; Chhetri, B.; Arman, H. D.; Larionov, O. V.; J. Am. Chem. Soc. 2016, 138, 2985; Uetake, Y.; Niwa, T.; Hosoya, T.; Org. Lett. 2016, 18, 2758.

19. Chen, X.-L.; Ai, B.-R.; Dong, Y.; Zhang, X.-M.; Wang, J.-Y.; Tetrahedron Lett. 2017, 58, 3646.

20. Thomas, C.; Carlson, R. E.; WO pat. 20130256162013.

21. Liu, D.; Ren, H.; Deng, L.; Zhang, T.; ACS Appl. Mater. Interfaces 2013, 5, 4937.

22. Yamamoto, T.; Ishibashi, A.; Koyanagi, M.; Ihara, H.; Eichenauer, N.; Suginome, M.; Bull. Chem. Soc. Jpn. 2017, 90, 604.

23. Wang, Z.; Sun, J.; Jia, X.; J. Polym. Sci., Part A: Polym. Chem. 2014, 52, 1962.

24. Guerrand, H. D. S.; Vaultier, M.; Pinet, S.; Pucheault, M.; Adv. Synth. Catal. 2015, 357, 1167.

25. Chen, K.; Zhang, S.; He, P.; Li, P.; Chem. Sci. 2016, 7, 3676; Minami, H.; Otsuka, S.; Nogi, K.; Yorimitsu, H.; ACS Catal. 2018, 8, 579 .

26. Wienhold, F.; Claes, D.; Graczyk, K.; Maison, W.; Synthesis 2011, 24, 4059.

27. Hu, J.; Sun, H.; Cai, W.; Pu, X.; Zhang, Y.; Shi, J. Z.; Org. Chem. 2016, 81, 14; Pinet, S.; Liautard, V.; Debiais, M.; Pucheault, M.; Synthesis 2017, 49, 4759.

28. Liu, S.; Zeng, X.; Xu, B.; Tetrahedron Lett. 2016, 57, 3706.

29. Bagutski, V.; Ros, A.; Aggarwal, V. K.; Tetrahedron 2009, 65, 9956.

30. Wozniak, A. A.; Jakubczyk, M.; Sporzynski, A.; Zukowska, G.; Inorg. Chem. Commun. 2011, 14, 1753.

31. Rhodes, C. N.; Brown, D. R.; J. Chem. Soc., Faraday Trans. 1993, 89, 1387.

32. Lennox, A. J. J.; Lloyd-Jones, G. C.; J. Am. Chem. Soc. 2012 , $134,7431$.

33. Yuen, A. K. L.; Hutton, C. A.; Tetrahedron Lett. 2005, 46, 7899.

Submitted: April 5, 2018

Published online: June 26, 2018 